\title{
Thoracic Epidural Blockade for Ventricular Tachycardia Storm in Patient with Takotsubo Cardiomyopathy
}

\author{
Jahagirdar Ashwini ${ }^{1}$, Makwana Durgesh², Date Girish ${ }^{3}$
}

\begin{abstract}
Introduction: Takotsubo cardiomyopathy is acute nonischemic myocardial dysfunction of the left and/or right ventricle which usually recovers completely within several days to weeks. We report a case where thoracic epidural analgesia was used to manage sympathetic storm in Takotsubo cardiomyopathy.

Case description: A 58-year-old diabetic female who was being treated for urinary tract infection and diabetic ketoacidosis for the past 2 days sustained an episode of pulseless ventricular tachycardia which was treated as per ACLS protocol. Troponin levels were raised, and 2D echocardiography was showing "Takotsubo cardiomyopathy" with typical apical ballooning of the left ventricle at the time of admission, and she was mechanically ventilated and receiving vasopressors. She continued to get episodes of ill-sustained ventricular tachycardia. In spite of conventional management, episodes of ill-sustained ventricular tachycardia continued, and hence, sympathetic blockade with thoracic epidural catheter was administered to control the ventricular tachycardia storm.

Conclusion: Sympathetic blockade to treat ventricular tachycardia is a promising approach which needs to be validated with more evidence. Keywords: Epidural anesthesia, Takotsubo cardiomyopathy, Ventricular tachycardia.

Indian Journal of Critical Care Medicine (2019): 10.5005/jp-journals-10071-23282
\end{abstract}

\section{INTRODUCTION}

Takotsubo cardiomyopathy is acute nonischemic myocardial dysfunction of the left and/or right ventricle which usually recovers completely within several days to weeks. ${ }^{1-3}$ Sympathetic overdrive and catecholamine excess play a central role in pathophysiology of Takotsubo cardiomyopathy. ${ }^{4-6}$ Sympathetic hyperactivity leads to life-threatening arrhythmias. Ventricular arrythmias account for about 2-10\% which significantly increases morbidity and mortality. ${ }^{7-9}$ Sympatholysis with thoracic epidural analgesia can be effective short-term measure to decrease arrhythmic burden. ${ }^{10-12}$ We report a case where thoracic epidural analgesia was used to manage sympathetic storm in Takotsubo cardiomyopathy.

\section{Case Description}

A 58-year-old diabetic female was transferred to our intensive care unit after undergoing an emergency coronary angiogram, which revealed normal coronaries. She was being treated for urinary tract infection and diabetic ketoacidosis for the past 2 days at a private hospital. During this period, she sustained an episode of pulseless ventricular tachycardia which was treated as per advanced cardiac life support protocol after which she got transferred for a coronary angiogram at our hospital. Troponin levels were raised and 2D echocardiography was showing left ventricular hypokinesia.

At the time of intensive cardiac unit admission, she was mechanically ventilated and receiving vasopressors (norepinephrine $0.18 \mu \mathrm{g} / \mathrm{kg} / \mathrm{minute}$ ). She was easily arousable to call and did not have any neurological deficit. She continued to get episodes of illsustained ventricular tachycardia. Her arterial blood gas revealed an optimal oxygenation and a high anion gap acidosis. Blood biochemistry showed a neutrophilic leukocytosis.

She was given intravenous amiodarone infusion along with correction of the existing electrolyte disturbances-hypokalemia (2.9 $\mathrm{mEq} / \mathrm{dL})$ and hypomagnesemia (1.2 mEq/dL). Intravenous \begin{tabular}{l}
\hline${ }^{1-3}$ Department of Critical Care Medicine, Sahyadri Super Speciality \\
Hospital, Pune, Maharashtra, India
\end{tabular}

Corresponding Author: Jahagirdar Ashwini, Department of Critical Care Medicine, Sahyadri Super Speciality Hospital, Pune, Maharashtra, India, Phone: +91 9822335330, e-mail: ashwini.jahagirdar@gmail.com How to cite this article: Ashwini J, Durgesh M, Girish D. Thoracic Epidural Blockade for Ventricular Tachycardia Storm in Patient with Takotsubo Cardiomyopathy. IJCCM 2019;23(11):529-532.

Source of support: Nil

Conflict of interest: None

meropenem was given in view of urosepsis. Human insulin infusion was administered for controlling blood sugar levels between 140 $\mathrm{mg} / \mathrm{dL}$ and $180 \mathrm{mg} / \mathrm{dL}$. Her general condition improved over the next 12 hours with resolution of shock, correction of acidosis, and restoration of a normal sinus rhythm. She was hence extubated. She was subjected to computed tomography urography which confirmed pyelonephritis.

Bedside 2D echocardiography was suggestive of "Takotsubo cardiomyopathy" with typical apical ballooning of the left ventricle. Speckle tracking on echocardiography suggested apical hypokinesia (Fig. 1).

Electrophysiologist opinion was sought and oral bisoprolol was added along with oral amiodarone. As she improved, she was shifted to the step-down unit for further management. Cardiac magnetic resonance imaging done to rule out structural abnormality revealed Takotsubo cardiomyopathy appearance in mid and apical cavity (Fig. 2).

Within 12 hours, she was shifted back to the intensive care unit for episodes of ill-sustained ventricular tachycardia with pulse. She was managed as per advanced cardiac life support guidelines with synchronized cardioversion. Intravenous antiarrhythmic bolus

(c) The Author(s). 2019 Open Access This article is distributed under the terms of the Creative Commons Attribution 4.0 International License (https://creativecommons. org/licenses/by-nc/4.0/), which permits unrestricted use, distribution, and non-commercial reproduction in any medium, provided you give appropriate credit to the original author(s) and the source, provide a link to the Creative Commons license, and indicate if changes were made. The Creative Commons Public Domain Dedication waiver (http://creativecommons.org/publicdomain/zero/1.0/) applies to the data made available in this article, unless otherwise stated. 

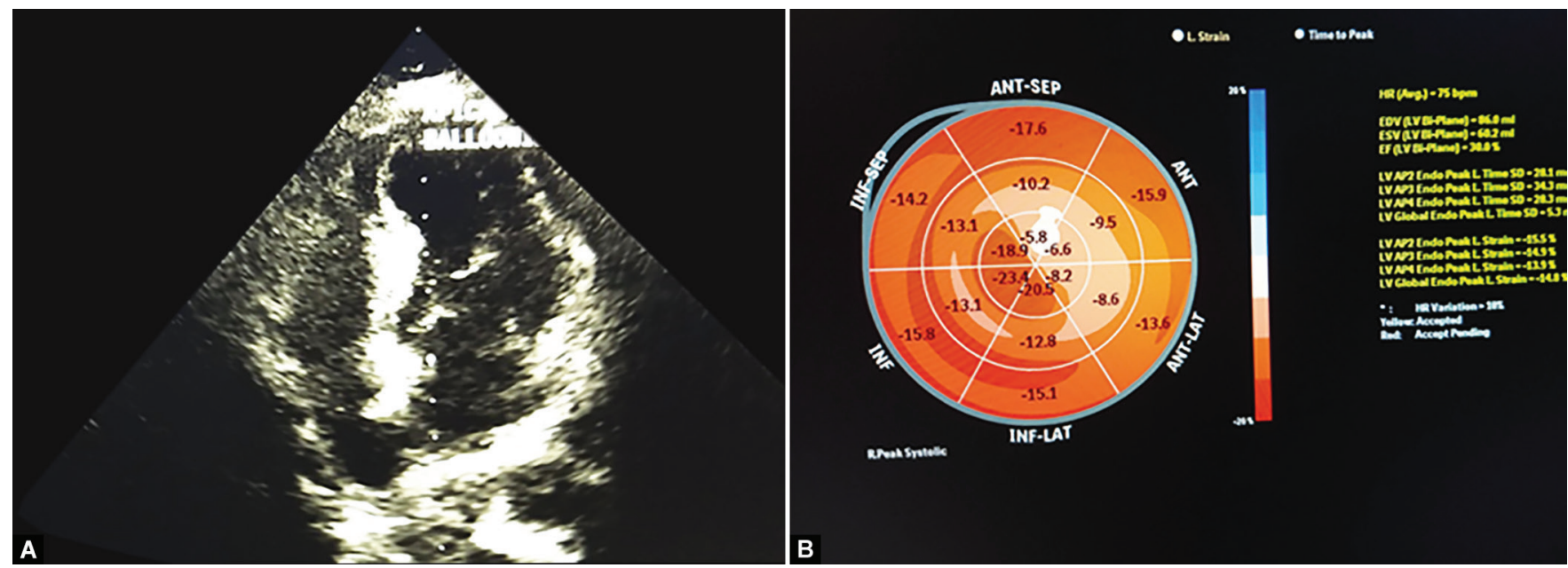

Figs $1 \mathrm{~A}$ and B: 2D echo suggestive of apical ballooning and speckle tracking echocardiography suggestive of decrease of motion of apical myocardial tissue
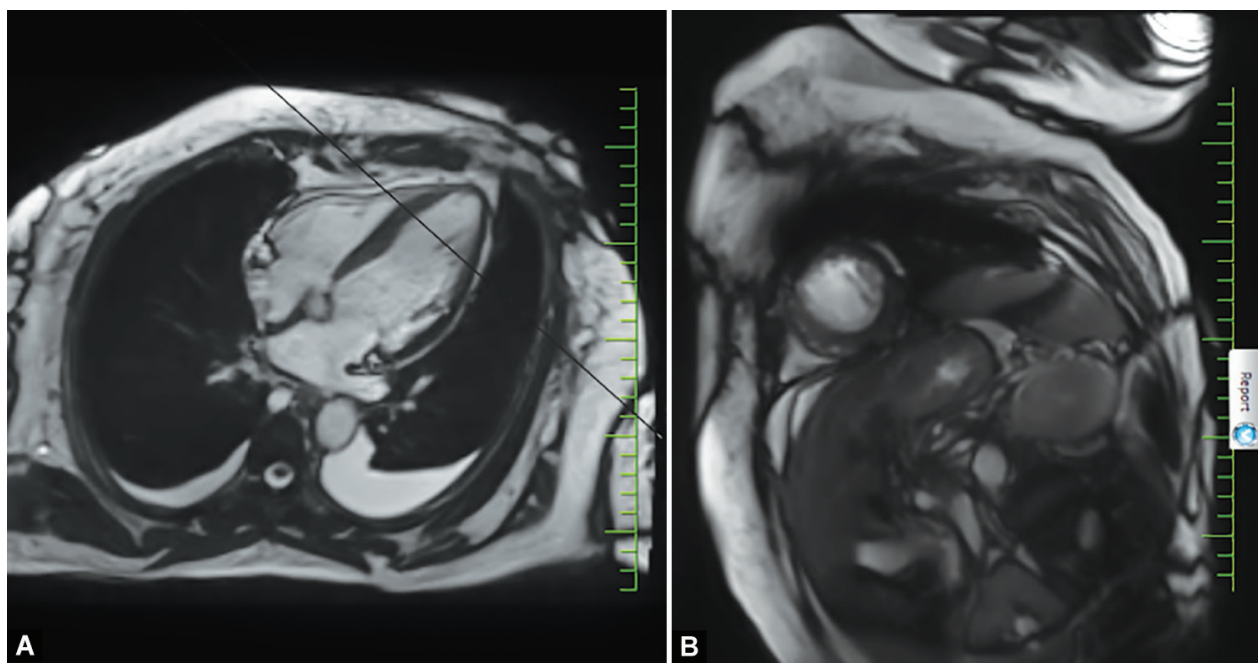

Figs 2A and B: Cardiac MRIT2 long axis four chamber, short axis two chamber and TRUFI coronal section suggestive of Takotsubo cardiomyopathy appearance in mid and apical cavity

doses of amiodarone and lignocaine were also tried. In spite of conventional management, episodes of ill-sustained ventricular tachycardia continued. Hence, it was decided to insert thoracic epidural catheter and administer sympathetic blockade to control the ventricular tachycardia storm and correct the metabolic derangements simultaneously. Hypokalemia $(3.2 \mathrm{mEq} / \mathrm{dL})$ found was the most likely cause of inciting the tachyarrhythmia.

After sympathetic blockade with ropivacaine $0.20 \%$ over the next 48 hours, no further episodes of ventricular tachycardia were recorded on Holter monitoring. She stabilized and epidural catheter was removed after 72 hours. The patient was discharged from the hospital at day 9 of admission on oral propefenone, amiodarone, and bisoprolol. Two dimensional echocardiography at discharge was suggestive of mild apical and distal interventricular septal hypokinesia, ejection fraction $45 \%$.

\section{Discussion}

The term "Takotsubo" is taken from a Japanese octopus trap with its characteristic shape which resembles the left ventricle in this particular type of cardiomyopathy. This is also called stress-related cardiomyopathy or broken heart syndrome. ${ }^{1-3,13-16}$ First described in 1990, it almost exclusively affects middle-aged females. Strong emotional stress has been found to be the precipitating event in many cases - hence the term "Broken heart syndrome." ${ }^{13-16}$ Many other prerequisites that have been cited for the diagnosis are as follows (Table 1). ${ }^{2}$

In this particular patient, the urosepsis along with diabetic ketoacidosis seems to be the precipitating event. The supportive investigations like a normal coronary angiogram, speckle tracking on the 2D echocardiography, elevated cardiac biomarkers, and cardiac magnetic resonance imaging help in proving our diagnosis.

The more challenging part was the refractory ventricular tachycardia associated with Takotsubo cardiomyopathy. ${ }^{4-9}$ The reported incidence of ventricular tachycardia with Takotsubo cardiomyopathy is $2-10 \%$. $^{7}$ The other common causes of ventricular tachycardia in the intensive care unit are hypoxia, infection, cardiac ischemia, catecholamine excess (endogenous or exogenous), or an electrolyte abnormality. They are usually amenable to management as per standard clinical protocols. In this particular patient, after trying the regular measures like metabolic corrections, drugs, and defibrillation, a novel method was tried in the form of sympathetic blockade by thoracic epidural analgesia. ${ }^{10-12}$ 
Table 1: Diagnosis criteria

1 Transient regional wall motion abnormalities of LV or RV myocardium which are frequently, but not always, preceded by a stressful trigger (emotional or physical).

2 The regional wall motion abnormalities usually ${ }^{\mathrm{a}}$ extend beyond a single epicardial vascular distribution, and often result in circumferential dysfunction of the ventricular segments involved.

3 The absence of culprit atherosclerotic coronary artery disease including acute plaque rupture, thrombus formation, and coronary dissection or other pathological conditions to explain the pattern of temporary LV dysfunction observed (e.g., hypertrophic cardiomyopathy, viral myocarditis).

$4 \quad$ New and reversible electrocardiography (ECG) abnormalities (ST-segment elevation, ST depression, LBBB $^{\text {b }}$, T-wave inversion, and/or QTc prolongation) during the acute phase (3 months)

5 Significantly elevated serum natriuretic peptide (BNP or NTproBNP) during the acute phase

6 Positive but relatively small elevation in cardiac troponin measured with a conventional assay (i.e., disparity between the troponin level and the amount of dysfunctional myocardium present) ${ }^{c}$

7 Recovery of ventricular systolic function on cardiac imaging at follow-up (3-6 months) ${ }^{\text {d }}$

${ }^{a}$ Acute, reversible dysfunction of a single coronary territory has been reported.

bLeft bundle branch block may be permanent after Takotsubo syndrome but should also alert clinicians to exclude other cardiomyopathies. T-Wave changes and QTc prolongation may take many weeks to months to normalize after recovery of LV function.

'Troponin-negative cases have been reported but are atypical.

${ }^{\mathrm{d}}$ Small apical infarcts have been reported. Bystander subendocardial infarcts have been reported, involving a small proportion of the acutely dysfunctional myocardium. These infarcts are insufficient to explain the acute regional wall motion abnormality observed.

Other options for treating ventricular storm are intracardiac defibrillator, general anesthesia, surgical left cardiac sympathetic denervation, and stellate ganglion block by percutaneous injection. ${ }^{10-12}$ In this particular patient, general anesthesia would have precipitated hemodynamic instability due to a poor left ventricular ejection fraction. Implantable defibrillator would have heightened the risk of infective endocarditis. Though epidural catheter insertion is contraindicated with active bloodstream infection, this was tried as the other options in the situation were not feasible. No complications related to procedure were seen.

Epidural sympathetic blockade for resistant ventricular tachycardia has been tried successfully with various local anesthetics and with various doses. We used ropivacaine in the strength of $0.20 \% .{ }^{17}$ Epidural catheter insertion was done at T6 level with tip at approximate T3 level and complete sympathetic blockade of segment T1-T4 was achieved. At initiation, 10-mL injection of ropivacaine, $0.20 \%$, was administered via the epidural catheter as bolus followed by an infusion at $3 \mathrm{~mL} /$ hour. The dose was titrated according to arrhythmic response. Studies have been done where cervical ganglion blockade was tried. ${ }^{18-21}$ Subsequent evidence suggests that similar results were possible even with high thoracic level block T1-T4. ${ }^{18-21}$ Epidural blockade was continued for 72 hours.

The postulated mechanism by which sympathetic blockade works is by decreasing cardiac sympathetic drive, increasing myocardial perfusion, and decreasing myocardial workload. ${ }^{10-12}$
A few case reports and a small case study have also shown successful epidural blockade for treating ventricular storm. ${ }^{22,23}$ The case report cited epidural blockade for treating ventricular tachycardia successfully in a patient with cardiac ischemia. The etiology for ventricular tachycardia in the aforementioned study was in patients with structural heart disease and implantable defibrillator. Patients underwent video assisted thoracoscopic surgical sympathetic blockade and were clinically followed-up for the recurrence of ventricular tachycardia. ${ }^{22}$ Such studies have been conducted on animal models also. ${ }^{24,25}$ We did not have any objective method of measuring the sympathetic tone.

Even though we were successful in our effort, more studies and evidence are needed to establish this as a recommended therapy. Complications reported are procedural complications associated with insertion or removal of the epidural catheter, epidural hematomas, infections, and hypotension.

\section{Conclusion}

Takotsubo cardiomyopathy is a unique condition which is mostly reversible. Rarely, it can get complicated with ventricular tachycardia. Resistant ventricular tachycardia has a high mortality. Sympathetic blockade to treat ventricular tachycardia is a promising approach which needs to be validated with more evidence.

\section{Declaration of Patient Consent}

The authors certify that they have obtained all appropriate patient consent forms. In the form, the patient has/have given his/her/their consent for his/her/their images and other clinical information to be reported in the journal. The patient understand that their names and initials will not be published and due efforts will be made to conceal their identity, but anonymity cannot be guaranteed.

\section{References}

1. Eitel I, von Knobelsdorff-Brenkenhoff $F$, Bernhardt $P$, Carbone I, Muellerleile K, Aldrovandi A, et al. Clinical characteristics and cardiovascular magnetic resonance findings in stress (Takotsubo) cardiomyopathy. JAMA 2011;306(3):277-286. DOI: 10.1001/ jama.2011.992.

2. Lyon AR, Bossone E, Schneider B, Sechtem U, Citro R, Underwood SR, et al. Current state of knowledge on Takotsubo syndrome: a position statement from the taskforce on Takotsubo syndrome of the Heart Failure Association of the European Society of Cardiology. Eur J Heart Fail 2016;18(1):8-27. DOI: 10.1002/ejhf.424.

3. Prasad A, Lerman A, Rihal CS. Apical ballooning syndrome (Takotsubo or stress cardiomyopathy): a mimic of acute myocardial infarction. Am Heart J 2008;155(3):408-417. DOI: 10.1016/j.ahj.2007. 11.008 .

4. Abraham J, Mudd JO, Kapur NK, Klein K, Champion HC, Wittstein IS. Stress cardiomyopathy after intravenous administration of catecholamines and beta-receptor agonists. J Am Coll Cardiol 2009;53(15):1320-1325. DOI: 10.1016/j.jacc.2009.02.020.

5. Paur H, Wright PT, Sikkel MB, Tranter MH, Mansfield C, O'Gara P, et al. High levels of circulating epinephrine trigger apical cardiodepression in a beta2-adrenergic receptor/Gi-dependent manner: a new model of Takotsubo cardiomyopathy. Circulation 2012;126(6):697-706. DOI: 10.1161/CIRCULATIONAHA.112.111591.

6. Wittstein IS, Thiemann DR, Lima JA, Baughman KL, Schulman SP, Gerstenblith G, et al. Neurohumoral features of myocardial stunning due to sudden emotional stress. N Engl J Med 2005;352(6):539-548. DOI: 10.1056/NEJMoa043046.

7. Stiermaier T, Eitel C, Denef S, Desch S, Schuler G, Thiele H, et al. Prevalence and clinical significance of life-threatening arrhythmias in 
Takotsubo cardiomyopathy. J Am Coll Cardiol 2015;65(19):2148-2150. DOI: 10.1016/j.jacc.2015.02.062.

8. Stiermaier T, Moeller C, Oehler K, Desch S, Graf T, Eitel C, et al. Longterm excess mortality in Takotsubo cardiomyopathy: predictors, causes and clinical consequences. Eur J Heart Fail 2016;18(6):650-656. DOI: 10.1002/ejhf.494.

9. Templin C, Ghadri JR, Diekmann J, Napp LC, Bataiosu DR, Jaguszewski $M$, et al. Clinical features and outcomes of Takotsubo (stress) cardiomyopathy. N Engl J Med 2015;373(10):929-938. DOI: 10.1056/ NEJMoa1406761.

10. Meissner A, Eckardt L, Kirchhof P, Weber T, Rolf N, Breithardt G, et al. Effects of thoracic epidural anesthesia with and without autonomic nervous system blockade on cardiac monophasic action potentials and effective refractoriness in awake dogs. Anesthesiology 2001;95(1):132-138. DOI: 10.1097/00000542-20010700000023.

11. Howard-Quijano K, Takamiya T, Dale EA, Yamakawa K, Zhou W, Buckley $U$, et al. Effect of thoracic epidural anesthesia on ventricular excitability in a porcine model. Anesthesiology 2017;126(6): 1096-1106. DOI: 10.1097/ALN.0000000000001613.

12. Bourke T, Vaseghi M, Michowitz Y, Sankhla V, Shah M, Swapna N, et al. Neuraxial modulation for refractory ventricular arrhythmias value of thoracic epidural anesthesia and surgical left cardiac sympathetic denervation. Circulation 2010;121(21):2255-2262. DOI: 10.1161/CIRCULATIONAHA.109.929703.

13. Eitel I, Stiermaier T, Graf T, Möller C, Rommel KP, Eitel C, et al. Optical coherence tomography to evaluate plaque burden and morphology in patients with Takotsubo syndrome. J Am Heart Assoc 2016;5(12):e004474. DOI: 10.1161/JAHA.116.004474.

14. Stiermaier T, Moller C, GrafT, Eitel C, Desch S, Thiele H, et al. Prognostic usefulness of the ballooning pattern in patients with Takotsubo cardiomyopathy. Am J Cardiol 2016;118(11):1737-1741. DOI: 10.1016/ j.amjcard.2016.08.055.

15. Sato HTHUT. Tako-tsubo-like left ventricular dysfunction due to multivessel coronary spasm. Clinical aspects of myocardial injury: from ischemia to heart failure. 1990. pp. 56-64.

16. Citro R, Bossone E, Parodi G, Carerj S, Ciampi Q, Provenza G, et al. Clinical profile and in-hospital outcome of Caucasian patients with
Takotsubo syndrome and right ventricular involvement. Int J Cardiol 2016;219:455-461. DOI: 10.1016/j.ijcard.2016.06.039.

17. Do DH, Bradfield J, Ajijola OA, Vaseghi M, Le J, Rahman S, et al. Thoracic epidural anesthesia can be effective for the short-term management of ventricular tachycardia storm. J Am Heart Assoc 2017;6(11):e007080. DOI: 10.1161/JAHA.117.007080.

18. Estes EH, Izlar H. Recurrent ventricular tachycardia: a case successfully treated by bilateral cardiac sympathectomy. Am J Med 1961;31: 493-497. DOI: 10.1016/0002-9343(61)90132-2.

19. Vaseghi M, Gima J, Kanaan C, Ajijola OA, Marmureanu A, Mahajan A, et al. Cardiac sympathetic denervation in patients with refractory ventricular arrhythmias or electrical storm: intermediate and longterm follow-up. Heart Rhythm 2014;11(3):360-366. DOI: 10.1016/ j.hrthm.2013.11.028.

20. Ajijola OA, Lellouche N, Bourke T, Tung R, Ahn S, Mahajan A, et al. Bilateral cardiac sympathetic denervation for the management of electrical storm. J Am Coll Cardiol 2012;59(1):91-92. DOI: 10.1016/ j.jacc.2011.09.043.

21. Pedersen CT, Kay GN, Kalman J, Borggrefe M, Della-Bella $P$, Dickfeld T, et al. EHRA/HRS/APHRS expert consensus on ventricular arrhythmias. Europace 2014;16(9):1257-1283. DOI: 10.1093/europace/ euu194.

22. Vaseghi M, Barwad P, Malavassi Corrales FJ, Tandri H, Mathuria N, Shah $\mathrm{R}$, et al. Cardiac sympathetic denervation for refractory ventricular arrhythmias. J Am Coll Cardiol 2017;69(25):1359-1362,. DOI: 10.1016/ j.jacc.2017.04.035.

23. Mahajan A, Moore J, Cesario DA, Shivkumar K. Use of thoracic epidural anesthesia for management of electrical storm: a case report. Heart Rhythm 2005;2(12):1359-1362. DOI: 10.1016/j.hrthm.2005.09.004.

24. Irie T, Yamakawa K, Hamon D, Nakamura K, Shivkumar K, Vaseghi M. Cardiac sympathetic innervation via middle cervical and stellate ganglia and antiarrhythmic mechanism of bilateral stellectomy. Am J Physiol Heart Circ Physiol 2017;312(3):H392-H405. DOI: 10.1152/ ajpheart.00644.2016.

25. Schwartz PJ, Stone HL. Left stellectomy in the prevention of ventricular fibrillation caused by acute myocardial ischemia in conscious dogs with anterior myocardial infarction. Circulation 1980;62(6):1256-1265. DOI: 10.1161/01.cir.62.6.1256. 